Proceedings of the Edinburgh Mathematical Society (2006) 49, 1-15 (C)

DOI:10.1017/S0013091504000975 Printed in the United Kingdom

\title{
HYPONORMAL OPERATORS, WEIGHTED SHIFTS AND WEAK FORMS OF SUPERCYCLICITY
}

\author{
FRÉDÉRIC BAYART AND ETIENNE MATHERON \\ Laboratoire Bordelais d'Analyse et de Géométrie, UMR 5467, Université Bordeaux 1, \\ 351 Cours de la Libération, 33405 Talence Cedex, France \\ (bayart@math.u-bordeaux1.fr; matheron@math.u-bordeaux1.fr)
}

(Received 20 September 2004)

\begin{abstract}
An operator $T$ on a Banach space $X$ is said to be weakly supercyclic (respectively $N$-supercyclic) if there exists a one-dimensional (respectively $N$-dimensional) subspace of $X$ whose orbit under $T$ is weakly dense (respectively norm dense) in $X$. We show that a weakly supercyclic hyponormal operator is necessarily a multiple of a unitary operator, and we give an example of a weakly supercyclic unitary operator. On the other hand, we show that hyponormal operators are never $N$-supercyclic. Finally, we characterize $N$-supercyclic weighted shifts.
\end{abstract}

Keywords: hypercyclic; supercyclic; hyponormal operators; weighted shifts; Kronecker sets

2000 Mathematics subject classification: Primary 47A16; 47B20; 43A46

\section{Introduction}

The dynamics of linear operators have been extensively studied in the last few years. In this paper, we are mainly concerned with the notion of supercyclicity. Let $X$ be a separable, infinite-dimensional complex Banach space. If $T$ is a continuous linear operator on $X$, the orbit of a set $E \subset X$ under $T$ is defined by

$$
\operatorname{Orb}_{T}(E)=\bigcup_{n \in \mathbb{N}} T^{n}(E)
$$

An operator is said to be supercyclic if there exists a vector $x \in X$ whose scaled orbit, namely $\mathbb{C O r b}_{T}(x)=\left\{\lambda y ; \lambda \in \mathbb{C}, y \in \operatorname{Orb}_{T}(x)\right\}$, is norm dense in $X$. The study of such operators was initiated by Hilden and Wallen $[\mathbf{9}]$, who showed that the adjoint of every unilateral weighted shift is supercyclic.

Several generalizations of supercyclicity were proposed recently. One of them replaces the single supercyclic vector $x \in X$ with a finite set of vectors: $T$ is said to be finitely supercyclic if there is a finite $F \subset X$ such that the set $\left\{\lambda T^{n} x ; x \in F, \lambda \in \mathbb{C}, n \in \mathbb{N}\right\}$ is dense in $X$. It was recently shown, however, that all finitely supercyclic operators are in fact supercyclic [13]. 
A more successful generalization of supercyclicity is the notion of $N$-supercyclicity, which was introduced in [6] and also studied in [3]. Observe that an operator $T \in \mathcal{L}(X)$ is supercyclic if and only if there exists a one-dimensional subspace of $X$ whose orbit under $T$ is dense in $X$. The operator $T$ is said to be $N$-supercyclic $(1 \leqslant N<\infty)$ if $X$ has an $N$-dimensional subspace with dense orbit. It is shown in [6] that, for every $N \geqslant 2$, there exist natural examples of $N$-supercyclic Hilbert-space operators that are not $(N-1)$-supercyclic.

Another possible generalization consists in looking at the weak topology of the Banach space $X$. The notion of supercyclicity makes sense in any topological vector space, in particular in the space $(X, w)$, and this gives rise to the class of weakly supercyclic operators: one just requires that the scaled orbit of some vector $x \in X$ is weakly dense in $X$. It is shown in $[\mathbf{1 7}]$ that weakly supercyclic operators may fail to be norm supercyclic.

The study of $N$-supercyclic or weakly supercyclic operators is very far from being complete. At the end of $[\mathbf{6}],[\mathbf{3}]$ and [17], several open questions are raised. We quote three of them.

Question 1.1 (Sanders [17]). Does there exist a weakly supercyclic hyponormal operator?

Question 1.2 (Feldman [6] and Bourdon et al. [3]). Can a pure hyponormal operator be $N$-supercyclic?

Question 1.3 (Feldman [6]). For $N \geqslant 2$, is there a bilateral weighted shift which is $N$-supercyclic but not $(N-1)$-supercyclic? If so, can we characterize $N$-supercyclic weighted shifts?

Questions 1.1 and 1.2 are reminiscent of two results of Kitai [11] (in the hypercyclic case) and Bourdon [2]: no hyponormal operator can be supercyclic. Question 1.3 seems interesting because $N$-supercyclic operators which appear in $[\mathbf{6}]$ or in $[\mathbf{3}]$ are constructed as direct sums of supercyclic operators, and it would be nice to obtain some other, less 'ad hoc' examples. Moreover, hypercyclic and supercyclic weighted shifts have already been characterized by Salas $[\mathbf{1 5}, \mathbf{1 6}]$, so it is natural to consider $N$-supercyclic case.

We solve completely these three questions, and the answers are sometimes surprising. We prove that a weakly supercyclic hyponormal operator has to be a multiple of a unitary operator, but we exhibit a unitary operator which is weakly supercyclic. On the other hand, hyponormal operators are never $N$-supercyclic, for any $N \geqslant 1$. Finally, we prove that an $N$-supercyclic weighted shift is necessarily supercyclic.

The paper is organized as follows. In $\S 2$, we prove a result relating the notions of $N$-supercyclicity and $N$-multicyclicity (see the definition below). Combined with the Berger-Shaw theorem (a basic fact in the theory of hyponormal operators), this result is used in $\S 3$, where Questions 1.1 and 1.2 are solved. Question 1.3 is solved in $\S 4$, the main tool here being the characterization of supercyclic weighted shifts given by Salas $[\mathbf{1 6}]$.

To conclude this introduction, we recall the following basic result of Ansari [1], which will be used twice in this paper:

If $T$ is a supercyclic operator, then all powers of $T$ are supercyclic, with the same supercyclic vectors. 
It is not known whether or not Ansari's theorem is still valid in the $N$-supercyclic setting; the problem is explicitly raised in [3]. The following lemma is a kind of substitute, which may help to solve it.

Lemma 1.4. Let $q$ be a positive integer, and let $E$ be a subset of $X$. For $j=1, \ldots, q$, put $A_{j}=\overline{\left\{T^{n} x ; x \in E, n=j(\bmod q)\right\}}$. If $\operatorname{Orb}_{T}(E)$ is dense in $X$, then each set $A_{j}$ has non-empty interior in $X$.

Proof. We will use the following elementary fact, first observed by Miller [12] in this context: if $A$ and $B$ are two closed subsets of $X$ such that $B$ has empty interior and $A \cup B=X$, then $A=X$. If $\operatorname{Orb}_{T}(E)$ is dense in $X$, we have $X=A_{1} \cup \cdots \cup A_{q}$. Therefore, we are able to extract a decomposition

$$
X=A_{j_{1}} \cup \cdots \cup A_{j_{m}}, \quad 1 \leqslant j_{1}<j_{2}<\cdots<j_{m} \leqslant q,
$$

which is minimal in the sense that there exists no decomposition of $X$ with fewer than $m$ sets $A_{j}$. Since $T^{k}$ has a dense range, one has $X=\overline{T^{k}(X)}=\overline{T^{k}\left(A_{j_{1}}\right)} \cup \cdots \cup \overline{T^{k}\left(A_{j_{m}}\right)}$ for any $k \in \mathbb{N}$. Moreover, it is straightforward to check that $\overline{T^{k}\left(A_{j}\right)} \subset A_{j+k}$, where $j+k$ is taken to be modulo $q$. Thus, we also have $X=A_{j_{1}+k} \cup \cdots \cup A_{j_{m}+k}$ for any $k \in \mathbb{N}$. Now, assume some $A_{l}$ has empty interior. If $k$ is chosen in such a way that $j_{1}+k=l(\bmod q)$, then, using the elementary fact mentioned above, we get a decomposition $X=A_{j_{2}+k} \cup$ $\cdots \cup A_{j_{m}+k}$ with less than $m$ sets $A_{j}$; this is a contradiction.

\section{2. $N$-supercyclicity and $N$-multicyclicity}

In this section, we consider complex topological vector spaces $X$ and $Y$, which may or may not be normed spaces. All topological notions will be relative to the given topologies. In particular, in the case of a Banach space equipped with the weak topology, 'supercyclic' means in this section the same as 'weakly supercyclic' in the rest of the paper.

Recall that an operator $S \in \mathcal{L}(Y)$ is said to be cyclic if there exists some vector $y \in Y$ such that the linear span of $\operatorname{Orb}_{S}(y)$ is dense in $Y$. The ' $N$-dimensional' analogue of cyclicity is the notion of $N$-multicyclicity.

Definition 2.1. Let $N$ be a positive integer. An operator $S \in \mathcal{L}(Y)$ is said to be $N$-multicyclic if there exist $N$ vectors $y_{1}, \ldots, y_{N}$ in $Y$ such that the linear span of $\left\{P(S) y_{m} ; 1 \leqslant m \leqslant N, P \in \mathbb{C}[z]\right\}$ is dense in $Y$.

The following result provides a link between $N$-supercyclicity and $N$-multicyclicity; it will be an essential tool in our study of the dynamics of hyponormal operators. Below, by a rational number of $\mathbb{T}$ we mean a complex number $\omega$ of the form $\omega=\mathrm{e}^{2 \mathrm{i} \pi r}$, where $r \in \mathbb{Q}$.

Proposition 2.2. Let $T \in \mathcal{L}(X)$, let $p$ be a positive integer, and let $\omega_{1}, \ldots, \omega_{p}$ be pairwise distinct rational numbers of $\mathbb{T}$. Assume that $T$ is $N$-supercyclic, for some positive integer $N$. Then the operator $S=\omega_{1} T \oplus \cdots \oplus \omega_{p} T$, acting on $Y=X^{p}$, is $N$-multicyclic. 
Proof. We first treat the case $N=1$, since it may be easier to follow and already contains the main ideas; moreover, this will be the only case needed in the proof of Theorem 3.4 below. So assume that $T$ is supercyclic, and let $x \in X$ be a supercyclic vector for $T$. We show that $\bar{x}=(x, \ldots, x) \in X^{p}$ is a cyclic vector for $S$. In other words, given $\bar{u}=\left(u_{1}, \ldots, u_{p}\right) \in X^{p}$ and neighbourhoods of $0, \mathcal{O}_{1}, \ldots, \mathcal{O}_{p} \subset X$, we have to find a polynomial $P$ such that $P(S) \bar{x}-\bar{u} \in \mathcal{O}_{1} \times \cdots \times \mathcal{O}_{p}$.

Let $\left(v_{1}, \ldots, v_{p}\right) \in X^{p}$ and neighbourhoods of $0, \mathcal{O}_{1}^{\prime}, \ldots, \mathcal{O}_{p}^{\prime} \subset X$ to be chosen later. Also let $q$ be a positive integer such that $\omega_{i}^{q}=1$ for $i=1, \ldots, p$. By Ansari's Theorem $[\mathbf{1}]$, all vectors $T^{j} x, j=1, \ldots, p$, are supercyclic for the operator $T^{q}$. Thus, one can find positive integers $n_{1}, \ldots, n_{p}$ with $n_{j}=j(\bmod q)$ and complex numbers $\lambda_{1}, \ldots, \lambda_{p}$ such that

$$
\lambda_{j} T^{n_{j}} x-v_{j} \in \mathcal{O}_{j}^{\prime}
$$

for all $j \in\{1 ; \cdots ; p\}$. Now let $P$ be the polynomial defined by

$$
P(z)=\lambda_{1} z^{n_{1}}+\cdots+\lambda_{p} z^{n_{p}} .
$$

Since $\omega_{i}^{n_{j}}=\omega_{i}^{j}$ for $i, j \leqslant p$, we have

$$
P(S)=\sum_{j} \lambda_{j} \omega_{1}^{j} T^{n_{j}} \oplus \cdots \oplus \sum_{j} \lambda_{j} \omega_{p}^{j} T^{n_{j}} .
$$

Viewing line vectors as column vectors for typographical simplicity, it follows that the vector $P(S) \bar{x}$ is given by

$$
P(S) \bar{x}=M_{\omega} \cdot\left(\lambda_{1} T^{n_{1}} x, \ldots, \lambda_{p} T^{n_{p}} x\right),
$$

where $M_{\omega}$ is the Vandermonde matrix

$$
M_{\omega}=\left(\begin{array}{cccc}
\omega_{1} & \omega_{1}^{2} & \cdots & \omega_{1}^{p} \\
\omega_{2} & \omega_{2}^{2} & \cdots & \omega_{2}^{p} \\
\vdots & \vdots & & \vdots \\
\omega_{p} & \omega_{p}^{2} & \cdots & \omega_{p}^{p}
\end{array}\right)
$$

Thus, setting $\bar{v}=\left(v_{1}, \ldots, v_{p}\right)$, we obtain

$$
P(s) \bar{x}-M_{\omega} \cdot \bar{v} \in M_{\omega} \cdot\left(\mathcal{O}_{1}^{\prime} \times \cdots \times \mathcal{O}_{p}^{\prime}\right) .
$$

Since the Vandermonde matrix $M_{\omega}$ is invertible, one can now choose $\bar{v}=\left(v_{1}, \ldots, v_{p}\right)$ and $\mathcal{O}_{1}^{\prime}, \ldots, \mathcal{O}_{p}^{\prime}$ in such a way that $M_{\omega} \cdot \bar{v}=\bar{u}$ and $M_{\omega} \cdot\left(\mathcal{O}_{1}^{\prime} \times \cdots \times \mathcal{O}_{p}^{\prime}\right) \subset \mathcal{O}_{1} \times \cdots \times \mathcal{O}_{p}$. This concludes the proof in the case $N=1$.

We now treat the general case, following the same ideas. Assume that $T$ is $N$ supercyclic, and let $E \subset X$ be an $N$-dimensional subspace such that $\operatorname{Orb}_{T}(E)$ is dense in $X$. Let $\left(x_{1}, \ldots, x_{N}\right)$ be a basis of $E$, and for $m \in\{1 ; \cdots ; N\}$, put $\bar{x}_{m}=\left(x_{m}, \ldots, x_{m}\right) \in$ $X^{p}$. We show that the linear span of $\left\{P(S) \bar{x}_{m} ; 1 \leqslant m \leqslant N, \mathbb{P} \in \mathbb{C}[z]\right\}$ is dense in $X^{p}$. Denoting this linear span by $F$, it is sufficient to show that $F$ has non-empty interior in $X^{p}$. 
Let $q \geqslant p$ be a positive integer such that $\omega_{i}^{q}=1$ for all $i \in\{1 ; \cdots ; p\}$, and let $A_{1}, \ldots, A_{q}$ be the sets defined in Lemma 1.4. If $M_{\omega}$ is the Vandermonde matrix introduced in the first part of the proof, then the set

$$
\mathcal{W}=\left\{\bar{u} \in X^{p} ; \exists \bar{v} \in A_{1} \times \cdots \times A_{p} M_{\omega} \cdot \bar{v}=\bar{u}\right\}
$$

has a non-empty interior in $X^{p}$, because $M_{\omega}$ is invertible and each $A_{j}$ has a non-empty interior in $X$. Therefore, it is sufficient to show that $\mathcal{W} \subset F$; in other words, given $\bar{v}=\left(v_{1}, \ldots, v_{p}\right) \in A_{1} \times \cdots \times A_{p}$, one can approximate $M_{\omega} \cdot \bar{v}$ by vectors of the form

$$
P_{1}(S) \bar{x}_{1}+\cdots+P_{N}(S) \bar{x}_{N}
$$

where $P_{1}, \ldots, P_{N}$ are polynomials. This is done exactly as in part 1 of the proof: each $v_{j}$ is approximated by vectors of the form $\lambda_{1 j} T^{n_{j}} x_{1}+\cdots+\lambda_{N j} T^{n_{j}} x_{N}$, where $n_{j}=j(\bmod q)$, and the polynomials $P_{m}(z)=\sum_{j} \lambda_{m j} z^{n_{j}}$ do the job.

\section{Remarks.}

(1) If the rational numbers $\omega_{1}, \ldots, \omega_{p}$ are not supposed to be pairwise distinct, then our proof does not work any more. In particular, we have not proved that if $T$ is $N$-supercyclic, then $T \oplus T$ is $N$-multicyclic. In the hypercyclicity setting, this problem is studied in [7], where it is proved that if $T$ is a hypercyclic operator, then $T \oplus T$ is cyclic if and only if $T$ satisfies the so-called hypercyclicity criterion (we refer the reader to [7] for the relevant definitions).

(2) It is also clear that our proof uses in an essential way the fact that the $\omega_{i}$ are rational numbers. It would be interesting to know what happens when the $\omega_{i}$ are not assumed to be rational.

\section{Dynamics of hyponormal operators}

In this section, all operators act on an infinite-dimensional Hilbert space.

\subsection{The Berger-Shaw theorem}

We recall that a bounded operator $T$ on a Hilbert space $H$ is said to be hyponormal if $\|T h\| \geqslant\left\|T^{*} h\right\|$ for all $h \in H$, which means that its self-commutator $\left[T^{*}, T\right]=T^{*} T-T T^{*}$ is a positive operator. Our reference on hyponormal operators is [4]. Bourdon's proof that a hyponormal operator is never supercyclic uses in a clever way some elementary estimations of the norms $\left\|T^{n} h\right\|, h \in H$. This method seems to be inefficient in the weakly supercyclic case. We replace it by a powerful result on $N$-multicyclic hyponormal operators: the so-called Berger-Shaw theorem.

Theorem 3.1. Let $S$ be a hyponormal operator. If $S$ is $N$-multicyclic for some positive integer $N$, then $\left[S^{*}, S\right]$ is a trace class operator, and

$$
\operatorname{Tr}\left(\left[S^{*}, S\right]\right) \leqslant \frac{N}{\pi} \operatorname{area}(\sigma(S)) .
$$


The exact value of the right-hand side is unimportant for the use we shall make of the Berger-Shaw theorem: we just need to know that $\operatorname{Tr}\left(\left[S^{*}, S\right]\right)$ is bounded by some constant $C(N,\|S\|)$ depending only on $N$ and $\|S\|$.

\subsection{Hyponormal operators and $N$-supercyclicity}

Let $N$ be a positive integer. It was proved by Feldman [6, Theorem 4.9] that a normal operator is never $N$-supercyclic. We extend this result to the case of hyponormal operators.

Theorem 3.2. Hyponormal operators are never $N$-supercyclic.

Proof. By Feldman's result mentioned above, it is sufficient to show that if $T \in \mathcal{L}(H)$ is hyponormal and $N$-supercyclic, then $T$ is normal. Let $p$ be any positive integer, and let $\omega_{1}, \ldots, \omega_{p}$ be the $p$ th roots of 1 . By Proposition 2.2, the operator $S=\omega_{1} T \oplus \cdots \oplus \omega_{p} T$ is $N$-multicyclic. Moreover, $S$ is hyponormal because $T$ is hyponormal. By the Berger-Shaw theorem, it follows that $\left[S^{*}, S\right]$ is a trace-class operator, and $\operatorname{Tr}\left(\left[S^{*}, S\right]\right) \leqslant C(N,\|S\|)$. Since $\|S\|=\|T\|$ and $\operatorname{Tr}\left(\left[S^{*}, S\right]\right)=p \operatorname{Tr}\left(\left[T^{*}, T\right]\right)$, we get

$$
0 \leqslant \operatorname{Tr}\left(\left[T^{*}, T\right]\right) \leqslant \frac{C(N,\|T\|)}{p} \text { for all } p \geqslant 1,
$$

so $\operatorname{Tr}\left(\left[T^{*}, T\right]\right)=0$, and hence $\left[T^{*}, T\right]=0$ because $\left[T^{*}, T\right]$ is a positive operator. Thus, $T$ is indeed a normal operator, and the proof is complete.

\subsection{Weakly supercyclic hyponormal operators}

In this subsection, we partly extend Bourdon's result that hyponormal operators cannot be supercyclic to the case of weakly supercyclic operators. Before stating our result, we prove the following lemma.

Lemma 3.3. Let $X$ be a Banach space, $X=X_{1} \oplus X_{2}$, and $S=S_{1} \oplus S_{2}$ with $S_{i} \in$ $\mathcal{L}\left(X_{i}\right), i=1,2$. Assume that there exists some positive number $r$ such that $\sigma\left(S_{1}\right) \subset \mathbb{D}_{r}$ and $\sigma\left(S_{2}\right) \subset \mathbb{C} \backslash \overline{\mathbb{D}}_{r}$, where $\mathbb{D}_{r}$ is the disc $\{|z|<r\}$. Then $S$ is not weakly supercyclic.

Proof. Since any non-zero multiple of a weakly supercyclic operator is weakly supercyclic, we may assume that $r=1$; thus, we have $\sigma\left(S_{1}\right) \subset \mathbb{D}$ and $\sigma\left(S_{2}\right) \subset \mathbb{C} \backslash \overline{\mathbb{D}}$. Suppose that $S$ is weakly supercyclic, and let $\left(f_{1}, f_{2}\right)$ be a weakly supercyclic vector. Since $\sigma\left(S_{2}\right) \subset \mathbb{C} \backslash \overline{\mathbb{D}}$, the operator $S_{2}$ is invertible and $\sigma\left(S_{2}^{-1}\right) \subset \mathbb{D}$. By the spectral radius formula, it follows that $\left\|S_{2}^{n} f_{2}\right\| \geqslant \beta C^{n}, n \in \mathbb{N}$, for some constants $\beta>0$ and $C>1$. By a result of Dilworth and Troitsky [5], this implies that the weak closure of the set $\left\{S_{2}^{n} f_{2} ; n \in \mathbb{N}\right\} \subset X_{2}$ does not contain 0 ; in other words, there exist continuous linear forms $\Phi_{1}, \ldots, \Phi_{p}$ on $X_{2}$ such that

$$
\forall n \geqslant 1, \quad \max _{1 \leqslant j \leqslant p}\left|\Phi_{j}\left(S_{2}^{n} f_{2}\right)\right| \geqslant 1 .
$$


Let us fix a non-zero linear functional $e^{*} \in X_{1}^{*}$. Let $\left(U_{k}\right)$ be the following sequence of weak open subsets of $X$ :

$$
U_{k}=\left\{\left(x_{1}, x_{2}\right) ;\left|e^{*}\left(x_{1}\right)-1\right|<1 / k \text { and } \max _{1 \leqslant j \leqslant p}\left|\Phi_{j}\left(x_{2}\right)\right|<1 / k\right\} .
$$

One can find an increasing sequence $\left(n_{k}\right)$ of integers and a sequence $\left(\lambda_{k}\right)$ of scalar numbers satisfying $\lambda_{k} S^{n_{k}}\left(f_{1}, f_{2}\right) \in U_{k}$. This implies

$$
\left|\lambda_{k}\right| \max _{1 \leqslant j \leqslant p}\left|\Phi_{j}\left(S_{2}^{n_{k}} f_{2}\right)\right| \leqslant 1 / k,
$$

hence $\lambda_{k}$ tends to 0 . Now, since $\sigma\left(S_{1}\right) \subset \mathbb{D}$, the operator $S_{1}$ is power bounded. Therefore, $\left|e^{*}\left(\lambda_{k} T_{1}^{n_{k}} f_{1}\right)\right|$ tends to 0 , which is a contradiction.

Now we can prove the following partial extension of Bourdon's result.

Theorem 3.4. Every weakly supercyclic hyponormal operator is a multiple of a unitary operator.

Proof. The proof will be carried out in two steps. In the first step, we reduce the problem to the case of weakly supercyclic normal operators. In the second step, we use Lemma 3.3 to show that such operators are necessarily multiples of unitary operators.

Step 1. A weakly supercyclic hyponormal operator is necessarily normal.

This is proved exactly as in Theorem 3.2, using Proposition 2.2 (with $N=1$ ) and the Berger-Shaw theorem. The only difference is that, in the present case, we apply Proposition 2.2 with $X=(H, w)$, the Hilbert space $H$ endowed with its weak topology, so we have to note that weak cyclicity (for the operators $S$ associated with $T$ ) is equivalent to norm cyclicity.

Step 2. A weakly supercyclic normal operator is a multiple of a unitary operator.

Let $T$ be a weakly supercyclic normal operator on the Hilbert space $H$. By the spectral theorem, we may assume that $T$ is a multiplication operator; this means that $T$ acts on $L^{2}(\Omega, \mu)$, for some finite measure space $(\Omega, \mu)$, and is defined by $T f=\phi f$, where $\phi \in L^{\infty}(\mu), \phi \neq 0$. We have to show that $|\phi|$ is constant $\mu$-almost everywhere. By contradiction, assume that this is not the case. Then one can find $r>0$ and $\varepsilon>0$ such that $A=\{z ;|\phi(z)|<r-\varepsilon\}$ and $B=\{z ;|\phi(z)|>r+\varepsilon\}$ have $\mu$-positive measure. Put $C=\Omega \backslash(A \cup B)$, and $d \mu_{A}=\mathbf{1}_{A} d \mu, d \mu_{B}=\mathbf{1}_{B} d \mu, d \mu_{C}=\mathbf{1}_{C} d \mu$. We can decompose $L^{2}(\mu)$ and $T$ as

$$
L^{2}(\mu)=L^{2}\left(\mu_{A}\right) \oplus L^{2}\left(\mu_{B}\right) \oplus L^{2}\left(\mu_{C}\right) \quad \text { and } \quad T=M_{\left.\phi\right|_{A}} \oplus M_{\left.\phi\right|_{B}} \oplus M_{\left.\phi\right|_{C}} .
$$

The operator $S=M_{\left.\phi\right|_{A}} \oplus M_{\left.\phi\right|_{B}}$ is weakly supercyclic because $T$ is, and this is impossible by Lemma 3.3 .

To conclude this section, we now use Lemma 3.3 to prove the existence of a 'weak supercyclicity circle'. In the case of norm supercyclicity, the existence of such a circle was proved by Herrero [8]. 
Proposition 3.5. Let $X$ be a Banach space. If $T \in \mathcal{L}(X)$ is weakly supercyclic, then there exists a circle $r \mathbb{T}$ such that every component of the spectrum of $T$ intersects $r \mathbb{T}$.

Proof. Assume that the conclusion is not true. For each $r \in I=\left[0 ; \infty\left[\right.\right.$, let $\mathbb{D}_{r}$ be the disc $\{|z|<r\}$. We first show that there exist some $r \in I$ and two components of $\sigma(T)$, say $K_{1}, K_{2}$, such that $K_{1} \subset \mathbb{D}_{r}$ and $K_{2} \cap \overline{\mathbb{D}}_{r}=\emptyset$. Indeed, otherwise for each $r \in I$, either all components of $\sigma(T)$ meet $\mathbb{C} \backslash \mathbb{D}_{r}$, or all components of $\sigma(T)$ meet $\overline{\mathbb{D}}_{r}$, and the two cases are exclusive because we assume that no circle $r \mathbb{T}$ meets each component of $\sigma(T)$. Let us denote by $A$ the set of all $r \in I$ satisfying the first property, and $B$ the set of all $r$ satisfying the second property. By compactness, $A$ and $B$ are closed subsets (in fact, subintervals) of $I$, they are non-empty ( $A$ contains $r=0$ and $B$ contains $r=\|T\|$ ), and they form a partition of $I$ : this contradicts the connectedness of $I$. So we have proved the existence of $r, K_{1}, K_{2}$ satisfying $K_{1} \subset \mathbb{D}_{r}$ and $K_{2} \cap \overline{\mathbb{D}}_{r}=\emptyset$. Notice that $r$ is positive because $\mathbb{D}_{0}=\emptyset$. We put $K_{3}=\sigma(T) \backslash\left(K_{1} \cup K_{2}\right)$. Then the compact sets $K_{1}, K_{2}$ and $K_{3}$ satisfy the following properties:

(i) $\sigma(T)=K_{1} \cup K_{2} \cup K_{3}$;

(ii) $K_{1}, K_{2}$ are non-empty;

(iii) $K_{1} \subset \mathbb{D}_{r}, K_{2} \subset \mathbb{C} \backslash \overline{\mathbb{D}}_{r}$;

(iv) $K_{1}, K_{2}$ and $K_{3}$ are pairwise disjoint.

By the Riesz decomposition theorem, we may write $X=X_{1} \oplus X_{2} \oplus X_{3}$, and $T=$ $T_{1} \oplus T_{2} \oplus T_{3}$, with $\sigma\left(T_{i}\right)=K_{i}, i=1,2,3$. We conclude as previously by applying Lemma 3.3 to $S=T_{1} \oplus T_{2}$.

\subsection{Unitary operators}

In view of Theorem 3.4, it seems to be very interesting to characterize those unitary operators which are weakly supercyclic (if there is any); or equivalently, to determine for which Borel measures $\mu$ on $\mathbb{T}$ the multiplication operator $M_{z}$ defined on $L^{2}(\mu)$ by $M_{z} f=z f$ is weakly supercyclic. Using some results on thin sets from harmonic analysis, we give a surprising example of a weakly supercyclic unitary operator. Then, for a large class of measures, including all measures which are not singular with respect to Lebesgue measure on $\mathbb{T}$, we show that $M_{z}$ is not weakly supercyclic.

Example 3.6. There exists a unitary operator which is weakly supercyclic.

The proof relies on the following lemma. Recall that a finite (positive) measure space $(\Omega, \mu)$ is said to be non-atomic if every measurable set $A \subset \Omega$ with positive measure has a measurable subset $B$ such that $0<\mu(B)<\mu(A)$. By the Lyapunov convexity theorem (see [14]), it follows that, for any measurable set $A \subset \Omega$, the range of $\mu_{\mid A}$ is the whole interval $[0 ; \mu(A)]$.

Lemma 3.7. Let $(\Omega, \mu)$ be a finite, non-atomic measure space, and let $\mathcal{F}$ be the set of all measurable functions $f: \Omega \rightarrow \mathbb{C}$ with constant modulus. Then $\mathcal{F}$ is weakly dense in $L^{2}(\mu)$. 
We postpone the proof of the lemma, and now give the promised example. Recall that a compact set $E \subset \mathbb{T}$ is said to be a Kronecker set if the characters of $\mathbb{T}$ are uniformly dense in $\mathcal{C}(E, \mathbb{T})$, the set of all continuous functions on $E$ with constant modulus 1. For further details on Kronecker sets and other thin sets from harmonic analysis, we refer the reader to [10]. It is well known, and easy to prove by Baire category arguments, that there exist perfect Kronecker sets. Let $E$ be such a set, and let $\mu$ be any continuous Borel probability measure with support $E$. Finally, let $T=M_{z}$ be the multiplication operator acting on $L^{2}(\mu)$. We show that the set $\mathcal{D}=\left\{\lambda z^{n} ; \lambda \in \mathbb{C}, n \in \mathbb{N}\right\}$ is weakly dense in $L^{2}(\mu)$, which means that the constant function $\mathbf{1}$ is a weak supercyclic vector for $T$. Since $E$ is a Kronecker set, it is also a Dirichlet set, which means that the constant function 1 a uniform limit (on $E$ ) of a sequence $z^{p_{k}}$, with $p_{k} \in \mathbb{N}$ and $p_{k} \rightarrow \infty$. Indeed, since $\mathcal{C}(E, \mathbb{T})$ has no isolated point, $\mathbf{1}$ is the uniform limit of a sequence $\left(z^{q_{k}}\right)$, where $q_{k} \in \mathbb{Z}$ and $\left|q_{k}\right|$ tends to $\infty$. Since $\mathbf{1}$ is real valued, we have $\left\|\mathbf{1}-z^{q_{k}}\right\|_{\infty}=\left\|\mathbf{1}-z^{-q_{k}}\right\|_{\infty}$, so we may assume that some subsequence $\left(p_{k}\right)$ of $\left(q_{k}\right)$ tends to $\infty$. Now, let $f: E \rightarrow \mathbb{T}$ be any continuous function. Writing $f=f \mathbf{1}$, using the fact that $E$ is a Kronecker set and choosing a sequence $\left(p_{k}\right)$ tending fast enough to $\infty$, we see that $f$ is the uniform limit of a sequence $\left(z^{n_{k}}\right)$, where the $\left(n_{k}\right)$ are non-negative integers. Therefore, the norm-closure of $\mathcal{D}$ in $L^{2}(\mu)$ contains all continuous functions $f: E \rightarrow \mathbb{C}$ with constant modulus. Now, since $E$ is a Kronecker set, it is totally disconnected; hence, any Borel subset of $E$ can be approximated in $\mu$-measure by a relatively clopen set. On the other hand, every Borel function $f: E \rightarrow \mathbb{C}$ with constant modulus can be approximated in the $L^{2}(\mu)$-norm by a finite sum $\sum_{i=1}^{p} c_{i} \mathbf{1}_{A i}$, where the $A_{i}$ are Borel sets and the $c_{i}$ have the same modulus. Approximating each $A_{i}$ by a clopen set and observing that the characteristic function of a clopen set is continuous, one obtains an approximation of $f$ by a continuous function with constant modulus. Thus, the norm closure of $\mathcal{D}$ in $L^{2}(\mu)$ contains all Borel functions on $E$ with constant modulus. Since $\mu$ is a continuous measure with support $E$, the measure space $(E, \mu)$ is non-atomic, so one can apply the lemma to conclude the proof.

Proof of Lemma 3.7. It is sufficient to prove the following claim.

Claim 3.8. Let $\varphi \in L^{\infty}(\mu)$. If $\left(A_{1}, \ldots, A_{n}\right)$ is a measurable partition of $\Omega$, then one can find $f \in \mathcal{F}$ such that $\|f\|_{2} \leqslant 2\|\varphi\|_{\infty}$ and $\int_{A_{i}} f \mathrm{~d} \mu=\int_{A_{i}} \varphi \mathrm{d} \mu$ for all $i$.

Indeed, once this is done, it follows that given any function $\varphi \in L^{\infty}(\mu)$ and any finite family of step functions $\left(h_{1}, \ldots, h_{k}\right)$, one can find $f \in \mathcal{F}$ with $\|f\|_{2} \leqslant 2\|\varphi\|_{\infty}$ such that $\int f h_{j} \mathrm{~d} \mu=\int \varphi h_{j} \mathrm{~d} \mu$ for all $j$ : one just needs to choose a partition $\left(A_{1}, \ldots, A_{n}\right)$ which is compatible with all functions $h_{j}$. Since one has a uniform estimate on $\|f\|_{2}$, this implies easily that each function $\varphi \in L^{\infty}(\mu)$ is in the weak closure of $\mathcal{F}$, and the lemma is proved. To prove the claim, we may obviously assume that $\mu$ is a probability measure, $\varphi \neq 0$ and all sets $A_{i}$ have positive measure. Set $\alpha_{i}=\int_{A_{i}} \varphi \mathrm{d} \mu$, and $K=2\|\varphi\|_{\infty}$. Then

$$
\max \left\{\frac{\left|\alpha_{i}\right|}{\mu\left(A_{i}\right)} ; 1 \leqslant i \leqslant n\right\} \leqslant\|\varphi\|_{\infty}<K,
$$

so one can find positive numbers $\delta_{1}, \ldots, \delta_{n}$ and complex numbers $K_{1}, \ldots, K_{n}$ such that $\left|K_{i}\right|=K, \delta_{i}<\mu\left(A_{i}\right)$ and $K_{i} \delta_{i}=\alpha_{i}$ for all $i$. 
Let $i \in\{1 ; \cdots ; n\}$. Since the measure space $(\Omega, \mu)$ is non-atomic and $\delta_{i}<\mu\left(A_{i}\right)$, one can use the Lyapunov convexity theorem to find a measurable partition $\left(B_{i}^{+}, \tilde{A}_{i}, B_{i}^{-}\right)$ of $A_{i}$ such that $\mu\left(\tilde{A}_{i}\right)=\delta_{i}$ and $\mu\left(B_{i}^{+}\right)=\mu\left(B_{i}^{-}\right)$: one first chooses $\tilde{A}_{i} \subset A_{i}$ such that $\mu\left(\tilde{A}_{i}\right)=\delta_{i}$, and next $B_{i}^{+} \subset A_{i} \backslash \tilde{A}_{i}$ such that $\mu\left(B_{i}^{+}\right)=\frac{1}{2}\left(\mu\left(A_{i}\right)-\delta_{i}\right)$.

Now, let $f: \Omega \rightarrow \mathbb{C}$ be defined on each set $A_{i}$ by $f(x)=K_{i}$ if $x \in \tilde{A}_{i}$ and $f(x)= \pm K$ if $x \in B_{i}^{ \pm}$. Then $f \in \mathcal{F},\|f\|_{2}=K=2\|\varphi\|_{\infty}$ and $\int_{A_{i}} f \mathrm{~d} \mu=K_{i} \delta_{i}=\int_{A_{i}} \varphi \mathrm{d} \mu$ for all $i$. This concludes the proof.

We have in fact proved the following result:

if $\mu$ is a continuous probability measure on $\mathbb{T}$ supported on a Kronecker set, then the multiplication operator $M_{z}$ acting on $L^{2}(\mu)$ is weakly supercyclic.

With a little extra work, one can exhibit a larger class of thin sets giving rise to weakly hypercyclic multiplication operators. Recall that a compact set $E \subset \mathbb{T}$ is said to be a Helson set if every continuous function on $E$ is the restriction of a function with absolutely convergent Fourier series. By standard duality arguments, $E$ is Helson if and only if there exists some finite constant $\alpha$ such that

$$
\|\nu\| \leqslant \alpha \sup _{n}|\hat{\nu}(n)|
$$

for all complex measures $\nu$ supported on $E$. The smallest such $\alpha$ is called the Helson constant of $E$; it is obviously not smaller than 1 . Kronecker sets are Helson with constant 1 , but the family of Helson-1 sets is much larger than the family of Kronecker sets.

Example 3.9. Let $\mu$ be a continuous probability measure on $\mathbb{T}$, and let $T=M_{z}$ be the multiplication operator acting on $L^{2}(\mu)$. Assume that the support of $\mu$ is Helson with constant 1 . Then $T$ is weakly supercyclic.

Proof. Let $E$ be the support of $\mu$. As before, it is sufficient to show that the weak (actually the norm) closure in $L^{2}(\mu)$ of the set

$$
\mathcal{D}=\left\{\lambda z^{n} ; \lambda \in \mathbb{C}, n \geqslant 1\right\}
$$

contains all continuous functions on $E$ with constant modulus 1. Let $f$ be such a function, and let $\nu=\bar{f} \mu$. Since $E$ is Helson-1, one can find a sequence of integers $\left(m_{k}\right)$ such that $\left|\hat{\nu}\left(m_{k}\right)\right|$ tends to $\|\nu\|=1$ as $k \rightarrow \infty$. Thus, one can find complex numbers $\alpha_{k}$ such that $\left|\alpha_{k}\right|=1$ and

$$
\lim _{k \rightarrow \infty} \int\left(\alpha_{k} z^{m_{k}} \bar{f}-1\right) \mathrm{d} \mu=0 .
$$

Since $g_{k}=\alpha_{k} z^{m_{k}} \bar{f}$ has constant modulus 1 , it follows that $g_{k}$ tends to 1 in the $L^{2}(\mu)$ norm, as can be seen by expanding $\left|g_{k}-1\right|^{2}$. And, since $\left|g_{k}-1\right|=\left|\alpha_{k} z^{m_{k}}-f\right|$, we conclude that $\alpha_{k} z^{m_{k}}$ tends to $f$ in norm. So we are almost done, except that the integers $m_{k}$ need not be positive.

To overcome this slight complication, we observe that there exist a sequence of positive integers $\left(p_{k}\right)$ tending to $\infty$ and a sequence $\left(\beta_{k}\right) \subset \mathbb{T}$ such that $\beta_{k} z^{p_{k}} \rightarrow 1$ in the 
$L^{2}(\mu)$-norm. Indeed, since $E$ is not reduced to a single point (because $\mu$ is continuous), one can find a continuous function $h$ on $E$ with constant modulus 1 which is not a multiple of (the restriction of) a character. As above, such a function is the norm limit of a sequence $\left(\alpha_{k}^{\prime} z^{m_{k}^{\prime}}\right)$, where $\left|\alpha_{k}^{\prime}\right| \equiv 1$ and $m_{k}^{\prime} \in \mathbb{Z}$. The sequence $\left(m_{k}^{\prime}\right)$ is unbounded because $h$ is not a multiple of a character. Then, by setting $\beta_{k}=\alpha_{k+1}^{\prime} / \alpha_{k}^{\prime}$, we see that the sequence $\beta_{k} z^{m_{k+1}^{\prime}-m_{k}^{\prime}}$ tends to 1 in norm, and by conjugating and passing to a subsequence if necessary, we may also assume that $p_{k}=m_{k+1}^{\prime}-m_{k}^{\prime}$ tends to $\infty$. Of course, $\left(p_{k}\right)$ tends to $\infty$ as fast as we want; in particular, we may assume that $n_{k}=p_{k}+m_{k} \geqslant 0$ for each $k$. Since the functions $\alpha_{k} z^{m_{k}}$ are uniformly bounded, $\alpha_{k} \beta_{k} z^{n_{k}}-\alpha_{k} z^{m_{k}}$ tends to 0 , so we finally find that $\alpha_{k} \beta_{k} z^{n_{k}}$ tends to $f$. This concludes the proof.

Our last example exhibits a family of measures for which the multiplication operator $M_{z}$ is not weakly supercyclic. Recall that a rational number of $\mathbb{T}$ is a complex number $\omega$ of the form $\omega=\mathrm{e}^{2 \mathrm{i} \pi r}, r \in \mathbb{Q}$.

Example 3.10. Let $\mu$ be a Borel probability measure on $\mathbb{T}$, and let $T=M_{z}$ be the usual multiplication operator acting on $L^{2}(\mu)$. Assume that the following condition is fulfilled: for each measurable set $A \subset \mathbb{T}$ such that $\mu(A)=1$, there exists a rational number $\omega \in \mathbb{T}, \omega \neq 1$, such that $A \cap \omega A \neq \emptyset$. Then the operator $T$ is not weakly supercyclic. This happens in particular if $\mu$ is not singular with respect to Lebesgue measure on $\mathbb{T}$.

Proof. Assume that $T$ is weakly supercyclic, and let $f \in L^{2}(\mu)$ be a weak supercyclic vector for $T$. By Ansari's theorem [1] $f$ is a weak supercyclic vector for all operators $T^{q}, q \geqslant 1$; in particular, $f$ is a (norm) cyclic vector for all $T^{q}$. This implies that, for each $q \geqslant 1$, one can find a sequence of polynomials $\left(R_{n q}\right)_{n \in \mathbb{N}}$ such that $R_{n q}\left(z^{q}\right) f(z)$ converges $\mu$-almost everywhere to $z f(z)$ as $n \rightarrow \infty$. Since there are only countably many $q$ and $f(z) \neq 0$ for $\mu$-almost every $z \in \mathbb{T}$ (otherwise $f$ would not be cyclic for $T$ ), it follows that there exists a measurable set $A \subset \mathbb{T}$ with $\mu(A)=1$, such that $R_{n q}\left(z^{q}\right) \rightarrow z$ pointwise on $A$ for all $q \geqslant 1$. By assumption, one can find a rational number $\omega \in \mathbb{T} \backslash\{1\}$ such that $A \cap \omega A \neq \emptyset$; let $a \in A \cap \omega A$, and put $b=\omega^{-1} a \in A$. Since $\omega$ is a rational number, one can choose an integer $q \geqslant 1$ such that $a^{q}=b^{q}$, and by definition of the set $A$, we get

$$
a=\lim _{n \rightarrow \infty} R_{n q}\left(a^{q}\right)=\lim _{n \rightarrow \infty} R_{n q}\left(b^{q}\right)=b,
$$

which is a contradiction. Therefore, $T$ is not weakly supercyclic.

Finally, if $\mu$ is not singular with respect to Lebesgue measure, then the above condition is fulfilled. Indeed, if $A \subset \mathbb{T}$ is a measurable set such that $\mu(A)=1$, then $A$ has positive Lebesgue measure. All rational translates of $A$ have the same Lebesgue measure, so they cannot be pairwise disjoint. Thus, there exist rational numbers $\omega_{1} \neq \omega_{2} \in \mathbb{T}$ such that $\omega_{1} A \cap \omega_{2} A \neq \emptyset$, and it is sufficient to put $\omega=\omega_{1}^{-1} \omega_{2}$.

A characterization of weakly supercyclic unitary operators seems difficult to obtain. A first step could be to solve the following problem. 
Question 3.11. Let $\mu$ be a probability measure on $\mathbb{T}$ such that $\hat{\mu}(n) \rightarrow 0$ as $|n| \rightarrow \infty$. Can $M_{z}$ be weakly supercyclic on $L^{2}(\mu)$ ?

Observe that a negative answer to this question cannot be obtained by the type of arguments used in Example 3.10. Indeed, it was proved by Rudin that there exist compact sets $K \subset \mathbb{T}$ which are independent (in the sense that if $\omega_{1}^{n_{1}} \times \cdots \times \omega_{p}^{n_{p}}=1$ with $\omega_{i} \in K$ pairwise distinct and $n_{i} \in \mathbb{Z}$, then $n_{i}=0$ for all $i$ ) but do carry probability measures whose Fourier coefficients vanish at $\pm \infty$. On the other hand, Körner and Kaufman have shown that there exist Helson-1 sets supporting distributions whose Fourier coefficients vanish at infinity; but it is well known that these distributions cannot be measures. All these facts are highly non-trivial (see [10]).

Measures $\mu$ such that $\hat{\mu}(n) \rightarrow 0$ as $|n| \rightarrow \infty$ are called Rajchman measures. It is well known that all complex measures absolutely continuous with respect to a given Rajchman measure are again Rajchman. It follows that if $\mu$ is a Rajchman measure and $f \in L^{2}(\mu)$, then $z^{n} f \rightarrow 0$ weakly in $L^{2}(\mu)$, and this implies in particular that no scaled orbit of $M_{z}$ can be weakly sequentially dense in $L^{2}(\mu)$. On the other hand, all weakly supercyclic operators known to us do have the stronger property that some scaled orbit is weakly sequentially dense. So we are naturally led to the following question.

Question 3.12. Does there exist a weakly hypercyclic operator on some Banach space $X$ such that no scaled orbit of $T$ is weakly sequentially dense in $X$ ?

\section{N-supercyclic weighted shifts}

In this section, we consider bilateral bounded weighted shifts acting on $X=\ell^{p}(\mathbb{Z})$, $1 \leqslant p<\infty$, or $c_{0}(\mathbb{Z})$; that is, operators $T$ defined by $T e_{n}=w_{n} e_{n-1}$, where $\left(w_{n}\right)$ is a bounded sequence of positive numbers. Needless to say, $\left(e_{n}\right)$ is the canonical basis of $X$.

Theorem 4.1. In the class of bilateral weighted shifts, $N$-supercyclicity is equivalent to supercyclicity.

Proof. Let $T$ be a bilateral weighted shift, with associated weight sequence $\left(w_{n}\right)$. By contradiction, assume that $T$ is not supercyclic, yet $N$-supercyclic for some $N \geqslant 2$. For all pairs $(i, n) \in \mathbb{Z} \times \mathbb{N}$, set

$$
\gamma_{i n}=w_{i+1} \times \cdots \times w_{i+n} .
$$

In other words, $\gamma_{i n}$ is defined by the identities

$$
\left\langle e_{i}^{*}, T^{n} x\right\rangle=\gamma_{i n} x_{i+n},
$$

where $e_{i}^{*}$ is the $i$ th coordinate functional. By a result of Salas [16], the fact that $T$ is not supercyclic can be expressed as follows: there exist $q \in \mathbb{N}$ and $\alpha>0$ such that, for any $n \in \mathbb{N}$,

$$
\max \left\{\frac{\gamma_{j-n n}}{\gamma_{h n}} ;|j|,|h| \leqslant q\right\} \geqslant \alpha .
$$


Since $T$ is $N$-supercyclic, there exist $N$ vectors $a(1), \ldots, a(N)$ in $X$ such that, if $E=$ $\operatorname{span}(a(1), \ldots, a(N))$, then $\overline{\operatorname{Orb}_{T}(E)}=X$. In particular, one can approximate the vector

$$
f=e_{-q}+\cdots+e_{0}+\cdots+e_{q},
$$

so one may find an increasing sequence of integers $\left(p_{k}\right)_{k \geqslant 1}$ and $N$ sequences of complex numbers $\left(u_{p_{k}}(m)\right), 1 \leqslant m \leqslant N$, such that

$$
\left\|u_{p_{k}}(1) T^{p_{k}} a(1)+\cdots+u_{p_{k}}(N) T^{p_{k}} a(N)-f\right\| \rightarrow 0
$$

as $k \rightarrow \infty$. From now on, we set $I=\left\{p_{k} ; k \geqslant 1\right\}$.

By (4.1), we may assume, passing to a subsequence if necessary, that there exist two integers $\boldsymbol{j}, \boldsymbol{h}$ with $|\boldsymbol{j}|,|\boldsymbol{h}| \leqslant q$, such that

$$
\frac{\gamma_{\boldsymbol{j}-n n}}{\gamma_{\boldsymbol{h} n}} \geqslant \alpha \quad \text { for all } n \in I .
$$

For each $n \in I$, let

$$
x^{n}=u_{n}(1) T^{n} a(1)+\cdots+u_{n}(N) T^{n} a(N) .
$$

For $m \in \mathbb{Z}$, let also

$$
a_{m}=\left(a_{m}(1), \ldots, a_{m}(N)\right) \in \mathbb{C}^{N} .
$$

By the definition of $\gamma_{i n}$, the $i$ th coordinate of $x^{n}$ is given by

$$
\begin{aligned}
\left\langle e_{i}^{*}, x^{n}\right\rangle & =\gamma_{i n}\left(u_{n}(1) a_{i+n}(1)+\cdots+u_{n}(N) a_{i+n}(N)\right) \\
& =\gamma_{i n} \tilde{\Theta}_{n}\left(a_{i+n}\right),
\end{aligned}
$$

where $\tilde{\Theta}_{n}$ is a linear form on $\mathbb{C}^{N}$ depending only on $n$. The key point in the proof is the following claim.

Claim 4.2. Set $\Theta_{n}=\gamma_{\boldsymbol{h} n} \tilde{\Theta}_{n}, n \in I$. Then $\Theta_{n}\left(a_{\boldsymbol{h}+n}\right) \rightarrow 1$ as $n \rightarrow \infty$, while $\Theta_{n}\left(a_{m}\right) \rightarrow$ 0 for each fixed $m>\boldsymbol{j}$. Finally, $\Theta_{n}\left(a_{m}\right) \rightarrow 0$ as $m \rightarrow \infty$, for each fixed $n \in I$.

Proof of Claim 4.2. Evaluating $x^{n}-f$ on $e_{\boldsymbol{h}}^{*}$, we get $\left|\Theta_{n}\left(a_{\boldsymbol{h}+n}\right)-1\right| \leqslant\left\|x^{n}-f\right\|$ by definition of $\Theta_{n}$. This proves the first part of the claim.

Before proving the second part, we observe that the following property holds true: for each integer $m>\boldsymbol{j}$, there exists a constant $C(m)$ such that

$$
\frac{\gamma_{\boldsymbol{h} n}}{\gamma_{m-n n}} \leqslant C(m) \text { for all } n \in I .
$$

Indeed, one can write

$$
\frac{\gamma_{\boldsymbol{h} n}}{\gamma_{m-n n}}=\frac{\gamma_{\boldsymbol{h} n}}{\gamma_{\boldsymbol{j}-n n}} \times \frac{w_{\boldsymbol{j}+1-n} \cdots w_{m-n}}{w_{\boldsymbol{j}+1} \cdots w_{m}}
$$

By (4.2), the first quotient is bounded by $1 / \alpha$, while the second is bounded by $\|w\|_{\infty}^{m-\boldsymbol{j}} /\left(w_{\boldsymbol{j}+1} \ldots w_{m}\right)$. This proves (4.3). 
We now prove the second part of the claim, so let us fix $m>\boldsymbol{j}$. If $n>q+m$, then, evaluating $x^{n}-f$ on $e_{m-n}^{*}$, we get $\left|\gamma_{m-n n} \tilde{\Theta}_{n}\left(a_{m}\right)\right| \leqslant\left\|x^{n}-f\right\|$. Using (4.3), it follows that

$$
\left|\gamma_{\boldsymbol{h} n} \tilde{\Theta}_{n}\left(a_{m}\right)\right| \leqslant C(m)\left\|x^{n}-f\right\|,
$$

which proves the second part of the claim.

The third part is obvious because $a(1), \ldots, a(N)$ belong to $c_{0}(\mathbb{Z})$.

To conclude the proof of the theorem, let us now fix a positive number $\delta<1 /(N+1)$. Using Claim 4.2, one can construct by induction an increasing sequence $\left(n_{k}\right) \subset I$ such that, on setting $b_{k}=a_{\boldsymbol{h}+n_{k}}$ and $\Phi_{k}=\Theta_{n_{k}}$, the vectors $b_{k} \in \mathbb{C}^{N}$ and the linear forms $\Phi_{k} \in\left(\mathbb{C}^{N}\right)^{*}$ satisfy the following properties:

$$
\left.\begin{array}{l}
\left|\Phi_{i}\left(b_{k}\right)\right|<\delta \quad \text { if } i \neq k \\
\left|\Phi_{k}\left(b_{k}\right)\right|>1-\delta
\end{array}\right\}
$$

Observe that, since

$$
\left|\Phi_{k}\left(b_{k}\right)\right|>1-\delta>N \delta>\sum_{\substack{i=1 \\ i \neq k}}^{N+1}\left|\Phi_{i}\left(b_{k}\right)\right|
$$

for all $k \in\{1 ; \cdots ; N+1\}$, the matrix $\left(\Phi_{i}\left(b_{k}\right)\right)_{i, k=1}^{N+1}$ is diagonally dominant, hence invertible. It follows that the vectors $b_{1}, \ldots, b_{N+1}$ are linearly independent in $\mathbb{C}^{N}$, which is impossible.

\section{References}

1. S. Ansari, Hypercyclic and cyclic vectors, J. Funct. Analysis 128 (1995), 374-383.

2. P. Bourdon, Orbits of hyponormal operators, Michigan Math. J. 44 (1997), 345-353.

3. P. Bourdon, N. Feldman and J. Shapiro, Some properties of $N$-supercyclic operators, Studia Math. 165 (2004), 135-157.

4. J. B. Conway, The theory of subnormal operators, Mathematical Surveys and Monographs, vol. 36 (American Mathematical Society, Providence, RI, 1991).

5. S. J. Dilworth And V. G. Troitsky, Spectrum of a weakly hypercyclic operator meets the unit circle, in Proc. Conf. Trends in Banach Spaces and Operator Theory, Memphis, TN, 2001, Contemporary Mathematics, vol. 321, pp. 67-69 (American Mathematical Society, Providence, RI 2003).

6. N. Feldman, $n$-supercyclic operators, Studia Math. 151 (2002), 141-159.

7. S. GRIVAux, Hypercyclic operators, mixing operators, and the bounded steps problem, J. Operat. Theory 54 (2005), 147-168.

8. D. A. Herrero, Limits of hypercyclic and supercyclic operators, J. Funct. Analysis 99 (1991), 179-190.

9. H. M. Hilden and L. J. Wallen, Some cyclic and non-cyclic vectors of certain operators, Indiana Univ. Math. J. 23 (1974), 557-565.

10. A. S. Kechris And A. Louveau, Descriptive set theory and the structure of sets of uniqueness, London Mathematical Society Lecture Notes Series, vol. 128 (Cambridge University Press, 1987). 
11. C. KitAI, Invariant closed sets for linear operators, PhD thesis, University of Toronto (1982).

12. V. G. MiLler, Remarks on finitely hypercyclic and finitely supercyclic operators, Integral Equations and Operator Theory 29 (1997), 110-115.

13. A. PerIs, Multi-hypercyclic operators are hypercyclic, Math. Z. 236 (2001), 779-786.

14. W. Rudin, Functional analysis (McGraw-Hill, New York, 1973).

15. H. Salas, Hypercyclic weighted shifts, Trans. Am. Math. Soc. 347 (1995), 993-1004.

16. H. Salas, Supercyclicity and weighted shifts, Studia Math. 135 (1999), 55-74.

17. R. Sanders, Weakly supercyclic operators, J. Math. Analysis Applic. 292 (2004), 148159 . 